\title{
CRÓNICA DEL CONGRESO INTERNACIONAL DE LA UNIVERSIDAD DE LA RIOJA «CONTRATACIÓN Y ARBITRAJE MERCANTIL INTERNACIONAL»
}

Jorge Julio EZQUERRO SOLAS

ABOGADO E INVESTIGADOR DE DERECHO MERCANTIL

UNIVERSIDAD DE LA RIOJA

Celebrándose en los días 5 y 6 de Noviembre de 2009, en el Salón de Actos del Edificio Politécnico de La Universidad de La Rioja, edificio moderno y dotado de todos los medios tecnológicos para el buen desarrollo y marcha del Congreso, tuvo lugar el "Congreso Internacional de Contratación y Arbitraje Mercantil Internacional”, organizado por la Universidad de La Rioja y promovido por proyecto del Ministerio de Ciencia e Innovación I+D+I (DER 2008-02244/JURI).

Compusieron su patrocinio la Fundación Cajarioja y el Consejo Regulador de la Denominación de Origen Rioja, y contó además, con el apoyo de la Comisión de las Naciones Unidas para el Derecho Mercantil Internacional (CNUDMI-UNCITRAL),el Instituto Internacional para la Unificación del Derecho Privado (UNIDROIT), el MOOT Madrid, el CISG Advisory Council y la Pace Law School.

El congreso contó con la colaboración de un Comité Científico de reconocido prestigio, compuesto por D. Manuel Olivencia Ruiz, D. Rafael Illescas Ortiz, D. Agustín Madrid Parra y D. Pedro de Pablo Contreras.

En cuanto a la organización académica del mismo decir que la dirección del Congreso fue compartida por Doña $\mathrm{M}^{a}$ del Pilar Perales Viscasillas Catedrática de Derecho Mercantil de la Universidad de La Rioja y Doña Susana Pérez Escalona, Profesora Contratada Doctor de Derecho Mercantil en la Universidad de La Rioja. Coordinaron el Congreso $\mathrm{D}^{a}$ María Teresa Cobo Sáenz y D. Jorge Julio Ezquerro Solas asistidos técnicamente por D. David Ramos Muñoz.

El aforo, dada la gran amplitud del salón de actos que acababa de ser puesto en funcionamiento, al igual que el resto del edificio, fue más que suficiente, creándose un ambiente acogedor y cómodo durante toda la duración del congreso para las personas que iban a asistir a un total de dieciséis ponencias, y una mesa redonda.

El congreso comenzó a las 9:00 h, momento en el cual se entregó la oportuna documentación y teniendo lugar la necesaria acreditación, el acto de apertura corrió a cargo del Magnífico Rector de la Universidad de La Rioja D. José María Martínez de Pisón Cavero. 
Seguidamente se ofreció la primera conferencia propiamente dicha, en la que D. Rafael Illescas Ortiz, Catedrático de Derecho Mercantil en la Universidad Carlos III de Madrid y Presidente de la UNCITRAL, transmitió su opinión jurídicamente fundamentada sobre el Convenio de la CNUDMI de 2008 y sobre las Reglas de Rotterdam de 2009 . Han recibido el nombre de Reglas de Rotterdam al haber sido en aquella ciudad holandesa donde se procedió a su firma en una conferencia diplomática, temas que se enmarcaban dentro del área de transporte internacional. Cabe subrayarse que este texto está pendiente de ratificación por parte de España, por lo que sería el primer país del mundo en ratificar dicho texto.

A continuación, y tras la oportuna pausa para el café, comenzó el Primer Panel, que llevó por título La Armonización Internacional del Derecho de Contratos: Cuestiones Generales, en el hubo cuatro ponencias, cerradas por un debate. La primera de ellas, estuvo a cargo de D. Jan Ramberg, Profesor Emérito de la Universidad de Estocolmo y versó sobre los efectos legales de la Convención de Viena de I980 sobre compraventa internacional (CISG) fuera de su ámbito de aplicación.

La segunda, que tenía por tema La Convención de Viena y su impacto en los Principios UNIDROIT y el Derecho Contractual Europeo, estuvo a cargo de D. Antonio María Manuel Morales Moreno, Catedrático de Derecho Civil de la Universidad Autónoma de Madrid.

Por su parte, la tercera conferencia de la mañana, fue protagonizada por D. Sergio Cámara Lapuente, Catedrático de Derecho Civil de la Universidad de La Rioja, y versó sobre los Principios de Derecho Contractual Europeo y sobre el Marco Común de Referencia, debatiendo sobre si se trataba de encubrir lo que no era si no un Código Civil europeo.

La última conferencia del primer panel que iba a cargo de D. Albert H. Kritzer, Profesor del Instituto para el Derecho Mercantil Internacional de la Universidad de Pace en Nueva York, tuvo que ser suspendida por imposibilidad de viajar a España por enfermedad. Tristemente, lamentamos comunicar el posterior fallecimiento del insigne profesor, producido a consecuencia de la referida enfermedad.

A final de cada una de estas conferencias, tuvo lugar un interesante debate de cierre de las mismas, en el que participaron todos los ponentes de la mañana respondiendo a las preguntas del público asistente.

Tras el correspondiente almuerzo, tuvo lugar la Presentación del Consejo Asesor en materia de compraventa internacional de mercancías (CISG-AC) a cargo de D. Eric E. Bergsten, Presidente del CISG-AC y antiguo secretario de la CNUDMI.

A las I6:0o h, del día 5 de Noviembre, comenzó el segundo panel, titulado Desafíos en la Interpretación del Derecho Uniforme del Comercio Internacional. Dentro del mismo, tuvieron lugar tres conferencias.

La primera de ellas, que trataba sobre la Cesión de créditos en el comercio internacional, y más concretamente sobre la incidencia que en ello habían tenido la convención de CNUDMI y los trabajos de UNIDROIT, fue impartida por Da. Marta García Mandaloniz, Profesora Titular de Derecho Mercantil en la Universidad Carlos III de Madrid.

Inmediatamente después, tuvo lugar la conferencia sobre la Interpretación e integración en los instrumentos del Derecho Uniforme del Comercio Internacional, ahondando especialmente en la CISG, la cual estuvo a cargo de D. Anselmo Martínez Cañellas, Profesor Titular de Derecho Mercantil en la Universidad de Las Islas Baleares. 
Finalmente, fue D. Sieg Biselen, Profesor de Derecho Privado en la Universidad de Sudáfrica, quien trató la polémica cuestión de la inclusión de condiciones generales en los contratos mercantiles internacionales.

Como ya se hizo con el anterior panel, se cerraron las conferencias con un debate en el que cada ponente dio su posición sobre los temas tratados. Tras otra parada para café, tuvo lugar el plato fuerte de la tarde, una Mesa Redonda sobre el futuro del Derecho de la Contratación Comercial Internacional, dirigido y moderado por Doña $\mathrm{M}^{\mathrm{a}}$ del Pilar Perales Viscasillas, Catedrática de Derecho Mercantil de la Universidad de La Rioja. En esta mesa redonda participaron D. Renaud Sorieul, Secretario de la CNUDMI, Director de la división de Derecho Mercantil Internacional, D. Alejandro M. Garro, Profesor en la Universidad de Columbia (Nueva York) y Observador en UNIDROIT, y D. Rafael Illescas Ortiz, Catedrático de Derecho Mercantil de la Universidad Carlos III de Madrid y Presidente de la CNUDMI, quien ya estuvo a cargo de la primera conferencia del congreso tras su inauguración. Esta mesa redonda se cerró con otro debate muy interesante y de actualidad. Ya por la noche, tuvo lugar la cena de gala con los ponentes.

La segunda sesión, el viernes 6 de Noviembre de 2009, comenzó por la mañana con el tercer panel, que llevó por título Aspectos Prácticos en la Aplicación del Derecho Uniforme del Comercio Internacional, compuesto por tres conferencias.

La primera estuvo a cargo de D. Michael Bridge, Profesor de Derecho en la London School of Economics y trató sobre la resolución de los contratos mercantiles internacionales y más en concreto sobre lo que en ellos se consideraba incumplimiento esencial.

La segunda de las conferencias de la mañana, titulada Fuerza Mayor y Hardship en la CISG, Principios UNIDROIT, Principios Lando y DCFR fue explicada por $\mathrm{D}^{\mathrm{a}}$.Ingeborg Schwenzer, Profesora de Derecho Privado en la Universidad de Basel, Suiza.

La tercera y última conferencia de la mañana, fue impartida por D. Hiroo Sono, Asesor del Ministerio de Justicia en asuntos civiles de Japón y Profesor Visitante en la Universidad de Hokkaido. Versó sobre el debate suscitado en cuanto a la aplicabilidad de la CISG a los contratos de software.

Como ya era costumbre, se cerró el panel con el oportuno debate, y tras el mismo, se efectúo la oportuna pausa para el café. Momento que fue aprovechado para que en un ambiente más relajados los ponentes y asistentes pudieran seguir debatiendo sobre los temas objeto de la jornada.

Hacia el medio día, comenzó el cuarto panel, titulado, Arbitraje Comercial Internacional, quizás uno de los más esperados por los asistentes. Estuvo compuesto por tres conferencias.

La primera, que trató sobre la forma escrita del convenio arbitral $y$ sus últimas novedades, fue explicada por D. Alejandro M. Garro, Profesor de Derecho de la Universidad de Columbia (Nueva York), el cual ya había intervenido en la mesa redonda del día anterior.

La segunda conferencia, versó sobre el derecho aplicable al arbitraje en el Comercio Internacional, y estuvo a cargo de D. Loukas Mistelas, Profesor Clive M. Schmitthoff de Derecho Mercantil Trasnacional y Arbitraje en la Universidad de Queen Mary (Reino Unido).

Para finalizar las conferencias del cuarto panel, tuvo lugar la tercera de ellas, titulada Remedios monetarios: una comparación entre el arbitraje comercial internacional y el 
arbitraje de inversiones, y fue dada por D. John Gotanda, Profesor de Derecho en la Universidad de Villanova (EEUU).

Sin más dilación, el último de los paneles fue cerrado con acostumbrado debate en el que tomaron parte todos los ponentes del mismo y tras el cual, tuvo lugar la comida y seguidamente, la lectura de comunicaciones.

Por la tarde, e inmediatamente después de la lectura de comunicaciones que estuvieron a cargo de profesores tanto nacionales como internacionales, se dictó la Conferencia de Clausura, titulada El arbitraje Internacional en España tras la ley de Arbitraje de 2003, a cargo del insigne D. Manuel Olivencia Ruiz, Catedrático Emérito de Derecho Mercantil en la Universidad de Sevilla y Delegado de España ante la CNUDMI. Para finalizar todo este magnífico congreso, de I7:30 a I8:०o, tuvo lugar el Acto de Clausura del Congreso a cargo de sus directoras, y de D ${ }^{a}$ Cristina Maiso Fernández, Subdirectora General de Justicia del Gobierno de la Rioja.

Como apunte final queremos destacar que la Universidad de La Rioja se convirtió durante los días 5 y 6 de noviembre en el centro de debate de los modernos y candentes temas del Derecho Mercantil Internacional, reuniendo a los máximos especialistas en la materia y con una amplia asistencia de profesores y profesionales del Derecho tanto nacionales como extranjeros. 